\title{
Estudio de la enfermedad mínima residual en el cáncer infantil
}

R. López Almaraz*, J. M. Raya Sánchez**, B. Martínez Pineda*, R. Cabrera Rodríguez*, J. Rodíguez Luis*

\section{Resumen}

El estudio de la enfermedad mínima residual (EMR), en distintas neoplasias infantiles, ha ido adquiriendo en los últimos años una importancia trascendental en la detección y seguimiento de pacientes con mayor probabilidad de recaída y como consecuencia, un mal pronóstico a largo plazo. Existen diferentes técnicas de estudio para determinar y cuantificar la EMR, según el tipo de neoplasia: la citometria de flujo, la inmunocitología y técnicas moleculares como la hibridación in situ con fluorescencia (FISH) y la transcripción inversa acoplada a la reacción en cadena de la polimerasa (RTPCR). En este artículo se repasan las diferentes técnicas utilizadas en busca de EMR durante y/o tras finalizar el tratamiento en leucemias agudas infantiles, neuroblastomas, rabdomiosarcomas y tumores de la familia del sarcoma de Ewing, así como su utilidad como factor pronóstico.

\section{Palabras clave:}

Enfermedad mínima residual. Citometría de flujo. Inmunocitología. RT-PCR. Leucemia aguda infantil. Neuroblastoma. Rabdomiosarcoma. Sarcoma de Ewing/ pPN ET.

Oncología, 2004; 27 (10):569-578

\footnotetext{
* Servicio de Pediatría (Unidad de 0 ncohematalogía Pediátrica).

** Servicio de Hematología y Hemoterapia.
} 


\section{Summary}

The study of minimal residual disease (MRD) in different childhood cancers has acquired a great importance in the last years for the detection and following up of patients with a high risk of relapse and the consequent long term poor prognosis. There are different techniques for MRD detection and quantification, depending on the tumor type: flow cytometry, immunocytology and biomolecular procedures - as fluorescence in situ hybridization (FISH) and reverse transcriptase-polymerase chain reaction (RTPCR)-. This work reviews the different techniques used to identify the MRD during and after treatment of childhood acute leukemia, neuroblastoma, rhabdomyosarcoma and Ewing's sarcoma, and their usefulness as prognostic factors.

Key words: Minimal residual disease. Flow cytometry. Immunocytometry. RTPCR. Childhood cancer. Acute leukemia. N euroblastoma. Rhabdomyosarcoma. Ewing's sarcoma (pN ET).

\section{Introducción}

En las últimas décadas, los avances en quimioterapia, radioterapia y en el trasplante de precursores hematopoyéticos han mejorado de forma considerable las posibilidades de supervivencia de los niños afectos de patología oncológica. Sin embargo, el riesgo de recurrencia continúa siendo, en muchos casos, un obstáculo importante para su curación. En este sentido, la detección de enfermedad mínima residual $(E M R)$ constituye un procedimiento de gran interés con objeto de adecuar los requerimientos terapéuticos' y además en algunos casos puede tener una clara trascendencia pronóstica ${ }^{1,2}$. El mayor conocimiento de la genética molecular en el cáncer infantil ha experimentado también progresos significativos. Los recientes avances de las técnicas de biología molecular han permitido la descripción de alteraciones cromosómicas en células tumorales y la identificación de oncogenes y genes supresores involucrados en la transformación maligna ${ }^{3-6}$, todo lo cual ha contribuido a mejorar los procedimientos diagnósticos, a desarrollar nuevos factores pronósticos y a planificar tratamientos más efectivos ${ }^{3,7}$.

La EMR consiste en la persistencia de un clon anormal, aún en niveles bajos, durante o tras finalizar el tratamiento. El inmunofenotipo y/o la citogenética y las técnicas moleculares pueden ser utilizadas para su estudio en leucemias y diferentes tumores sólidos infantiles. La EMR tiene significado pronóstico ya que puede predecir la recaída de la enfermedad y por este motivo, conocer su presencia nos puede ayudar a plantear estrategias terapéuticas para prevenirla ${ }^{6-11}$.

\section{Técnicas de estudio de la enfermedad mínima residual}

Los métodos de estudio para detectar EMR deben cumplir unos requisitos previos para poder ser considerados útiles. Dichos requisitos incluyen una elevada sensibilidad, especificidad, reproducibilidad y aplicabilidad de la técnica. Los procedimientos que actualmente cumplen estos requisitos son la inmunofenotipificación por citometría de flujo, la inmunocitología y la técnica de la reacción en cadena de la polimerasa (PCR) en tiempo real o real-time PCR. En el momento actual el mayor conocimiento, tanto de los marcadores inmunológicos (inmunofenotipo), con la utilización de una amplia batería de anticuerpos monoclonales, así como de las alteraciones cromosómicas analizadas a través de técnicas de citogenética o biología molecular (FISH y RT-PCR), han permitido profundizar en el concepto de remisión en diferentes cánceres infantiles mediante el estudio de la EM R-9.9. 12 .

Las técnicas más comúnmente utilizadas dependiendo de cada caso son:

\section{- Citometría de flujo}

Tecnología utilizada para analizar y definir el perfil inmunofenotípico de las células neoplásicas y establecer así la presencia de fenotipos aberrantes. Se utiliza principalmente en leucemias. Se basa en la aplicación de anticuerpos monoclonales específicos, dirigidos contra proteínas de membrana o intracitoplasmáticas, que llevan apareado un fluorocromo para su detección y visualización mediante un sistema informático apropiado. Posee una sensibilidad superior a $1 \times 10^{-4}$, es 
decir, es capaz de detectar una célula tumoral entre 10.000 células normales ${ }^{9,13-15}$.

\section{- Inmunocitología}

El objetivo de esta técnica es la identificación de estructuras antigénicas que permitan detectar células neoplásicas en un tejido (médula ósea, sangre periférica y/ o productos de aféresis), mediante anticuerpos monacionales "tumor-específicos". Se utiliza principalmente en el neuroblastoma. Posee una sensibilidad de $1 \times 10^{-516}$.

\section{- FISH (Hibridación in situ con fluorescencia)}

Técnica molecular que utiliza sondas de ADN marcadas con fluorocromos, que ponen de manifiesto la presencia o ausencia de un determinado fragmento genético, así como su localización cromosómica. Mediante un microscopio de fluorescencia se capta la imagen de la señal y la célula. Esta metodología puede aplicarse tanto en núcleos interfásicos como directamente en cromosomas. Puede hallar alteraciones cromosómicas específicas muy por debajo del nivel de detección de los estudios citogenéticos de bandeo tradicionales (cariotipo) $3,6,8$.

\section{- RT-PCR (Trascripción inversa acoplada a la reacción en cadena de la polimerasa)}

La PCR es una técnica molecular que permite amplificar secuencias específicas de ADN O ARN expresadas en las células tumorales, multiplicando por 100 el número de copias que podemos obtener de un determinado fragmento de ADN. Con la RT-PCR, se puede analizar el ARN de transcripción expresado en dichas células. Esto nos permite el estudio de los genes alterados por traslocociones primarias y de otros genes característicos asociados a determinados tipos de tumor. En la monitorización de la EMR es importante la cuantificación de ésta mediante la RT-PCR en tiempo real, de manera que la comparación de su nivel de amplificación con los estándares adecuados, proporciona una medida cuantitativa del grado de afectación. Posee una sensibilidad que se sitúa entre $1 \times 10^{-5}$ y 1 x $10^{-6} 6,8,16$.

\section{Detección de la EMR en las leucemias agudas infantiles}

La definición convencional de remisión completa morfológica en la leucemia aguda infantil implica una blastosis inferior al $5 \%$ en una médula ósea normocelular y la recuperación hematológica completa en sangre periférica. Este procedimiento clásico, si bien muy útil y en plena vigencia, es poco sensible para detectar enfer- medad residual. M ediante el empleo de las actuales técnicas de citometría de flujo y PCR para determinar $E M R$, que tienen una sensibilidad 100 veces superior a la citomorfología, es posible identificarla en numerosos pacientes en remisión. La introducción de estas técnicas ha conducido a una nueva definición de remisión en las leucemias según la cual podría considerarse remisión hematológica cuando no se detecta EM R con un umbral de $1 \times 10^{-4}(0,01 \%$ de células leucémicas del total de células nucleadas de la médula osea $)^{10,12,15,17,18}$.

Los dos marcadores moleculares más empleados en la detección de EMR en las leucemias, mediante PCR, son: a) el análisis de los reordenamientos clonales de los genes de las inmunoglobulinas (Ig) y del receptor de célula T(TCR), y b) la detección de aberraciones cromosómicas en las que se conocen los oncogenes implicados que dan lugar a regiones quiméricas de fusión características (por ejemplo el BCR/ ABL) ${ }^{19-23}$.

Algunas cuestiones relativas a la EMR en la leucemia aguda linfoblástica (LA L) son motivo de discusión en la actualidad, pero poco a poco se van acercando posturas.

¿En qué momento es útil realizar la determinación de EMR?

Cada vez existe mayor consenso en que sería deseable realizar el estudio de EM $R$ en la $L A L$ al final de la inducción, al final de la consolidación, a los 12 meses de tratamiento y tras finalizarlo, pues la detección de EMR nos aporta una valiosa información sobre la predicción del curso clínico de la enfermedad y nos permitiría adoptar actitudes terapéuticas en consecuencia $^{1,11-13,15,18}$

¿Existe relación demostrada entre la persistencia de EM R y el riesgo de recaída de la enfermedad?

Diversos trabajos realizados con análisis de citometría de flujo ponen de manifiesto que el riesgo de recidiva en los pacientes con EMR $\geq 1 \times 10^{-2}$ al finalizar la inducción es superior al $60 \%$ en el curso de los tres años siguientes, frente a menos del $15 \%$ en los que no se detecta EMR. Los pacientes que mantienen EMR con niveles $\geq 1 \times 10^{-3}$ después del tratamiento de consolidación tienen un riesgo de recidiva de aproximadamente un 70\%13-14. 0 tros autores han realizado estudios similares midiendo la EMR con las técnicas mencionadas de PCR y los resultados han sido superponibles: la probabilidad de recaída fue del $100 \%$ con niveles de EMR $\geq 1 \times 10^{-3}$ al finalizar la inducción (día + 36) comparada con un $14 \%$ con niveles menores $(p<0,001)^{24}$.

La detección de EMR por PCR en niños afectos de LA L-B y LA L-T, analizada en diferentes períodos del tratamiento (al finalizar la inducción y antes de co- 


\section{R. López Almaraz y cols.}

menzar la consolidación) y considerando un alto riesgo de recaída con niveles de EMR $\geq 1 \times 10^{-3}$, pone de manifiesto que en la LAL-T es más frecuente la detección de EMR y con cifras más elevadas, lo que refleja una mayor resistencia al tratamiento y peor pronóstico en este grupo de pacientes ${ }^{25,26}$. Recientemente se ha comunicado un estudio en el que se analiza la importancia de la detección de EMR por PCR a mayor largo plazo (segundo año del tratamiento), y los autores concluyen que la combinación de los hallazgos de EMR en los meses 1 y 24 tras el diagnóstico, pueden predecir con bastante fiabilidad la evolución clínica de los niños con LA L27.

El criterio diagnóstico de recaída meníngea en la $L A L$ es la presencia, en líquido cefalorroquídeo (LCR), de más de 5 leucocitos de morfología blástica por $\mathrm{mm}^{3}$, en ausencia de una punción lumbar traumática. Nos pueden surgir dudas de si un paciente presenta una meningiosis leucémica con un contaje bajo de leucocitos en el LCR. Para superar esto se recomienda realizar estudio de citometría de flujo ${ }^{28}$ o por $P C^{29}$ en el propio LCR que sometido a centrifugación presenta un contaje bajo de células en el examen citomorfológico. Si por cualquiera de estos métodos se detectara EM R a nivel del sistema nervioso central habría que intensificar el tratamiento sobre el mismo hasta la normalización permanente del $\mathrm{LCR}^{28,29}$.

En la leucemia aguda no linfoblástica o mieloblástica (LAN L), la detección de EMR depende principalmente de la detección de inmunofenotipos aberrantes mediante citometría de flujo, pues menos de un tercio de los pacientes presentan marcadores genéticos en su monitorización por técnicas moleculares ${ }^{8,30}$. Se está utilizando la RT-PCR para la detección del gen de fusión AML1-ETO que identifica la traslocación $\mathrm{t}(8 ; 21)(\mathrm{q} 22 ; q 22)$ asociada a la LAN L-M 2 , como control de la remisión en este subtipo leucémico. Todos los pacientes que tuvieron una remisión prolongada y sin recaída presentaron estudios con PCR negativos. Una EMR negativa detectada con la técnica de RTPCR antes de la consolidación predice un buen pronóstico ${ }^{20}$ y este hallazgo pudiera ayudar a cambiar el tipo de consolidación en pacientes con LANL con $t(8 ; 21)^{21}$.

Esta técnica también puede detectar EMR en la LAN L-M ${ }_{4}$ Eo, subtipo que se caracteriza por la presencia del gen de fusión CBF $\beta$-MYH11 que identifica la inversión inv(16)(p13;q22). Todos los pacientes en remisión completa inmediatamente después de la terapia de inducción y/ o consolidación tuvieron estudios por PCR positivos. Sin embargo, los que fueron negativos en el seguimiento posterior se mantuvieron en remisión completa durante más de 12 meses. El número de co- pias del gen CBF $\beta$-MYH11, tras la inducción y consolidación, fueron significativamente más altas en los que recayeron comparado con los que mantuvieron remisión completa, lo que confiere a esta determinación de EM R un importante valor predictivo 22,23 .

El éxito de los trasplantes de progenitores hematopoyéticos (TPH) en leucemias infantiles, tanto alogénicos como autólogos, depende de la persistencia o reaparición de EMR en el paciente trasplantado ${ }^{31-34}$. Estudios retrospectivos muestran que la presencia de EMR en $L A L$, analizada previamente a un TPH, se asocia a un alto riesgo de recaída posterior ${ }^{31}$. En este sentido, en un trabajo reciente que incluía pacientes afectos de $L A L$ que recibieron un trasplante alogénico de médula ósea, se realizó monitorización de EMR por citometría de flujo antes de comenzar el acondicionamiento y en los días +30 , +60 , +90 postrasplante y posteriormente cada 3 meses. Se vio que en pacientes con EM R positiva la supervivencia libre de enfermedad (SLE) fue del $33,3 \%$ comparada con el $73,5 \%$ de aquellos en los que era negativa. A simismo, durante el seguimiento, el incremento de los niveles de EMR precedió a la recaída medular en el $100 \%$ de los casos en el plazo de 1 a 6 meses $^{33}$. Similares resultados se observan en otro estudio que analiza la presencia o no de EMR postrasplante alogénico en 32 pacientes (23 niños y 9 adultos) con $L A L$, utilizando marcadores de los reordenamientos clonales del TCR y de la lg. Del total de pacientes, nueve presentaron EMR positiva, de los cuales 8 recayeron con una media de 5,5 meses postrasplante (rango de 0,5-30 meses). De los 23 pacientes con EMR negativa, recayeron seis. Nuevamente se demuestra que la presencia y sobre todo el aumento del nivel de EM R postrasplante aumenta el riesgo de recaída $a^{34}$.

Teniendo en cuenta todo lo anteriormente expuesto, ¿deben tomarse actitudes específicas en la práctica clínica diaria en función de los resultados de detección de EMR?

En la actualidad existen estudios en curso que utilizan la detección de EMR para estratificar a los pacientes en grupos de mayor o menor riesgo. Así, por ejemplo, el PETHEMA LA L-BR/ 2001 contempla cambiar los niños de protocolo terapéutico de bajo riesgo a alto riesgo, si por citometría de flujo se detecta EMR al $\geq$ $1 \%$ después del tratamiento de inducción $0>0,1 \%$ al finalizar el tratamiento de consolidación ${ }^{35}$. Los resultados de estos ensayos aportarán mayor claridad al papel de la EMR. Por otra parte, son necesarios más estudios para afirmar que con niveles de EMR $<0,01 \%$ tras la inducción los pacientes puedan curarse con tratamientos menos intensivos, lo que reduciría el riesgo de efectos secundarios a largo plazo 26, 34-38. 


\section{Detección de EMR en el neuroblastoma}

El neuroblastoma (N B) es el tumor sólido extracraneal más frecuente en la infancia. Se trata de una neoplasia muy heterogénea cuyo pronóstico en el momento del diagnóstico se correlaciona con la edad del paciente, el estadio de la enfermedad, las características biológicas y alteraciones genéticas múltiples del tumor. La quimioterapia mieloablativa seguida del trasplante autólogo de progenitores hematopoyéticos de sangre periférica (TASPE), se utiliza en los pacientes de alto riesgo que han alcanzado una remisión completa 0 muy buenas remisiones parciales tras la quimioterapia de inducción y la posterior cirugía del tumor primario. Sin embargo, el $40-50 \%$ de estos pacientes experimentan una recaída después del TASPE, resultando en este grupo la tasa de supervivencia libre de enfermedad a los 2 años, menor al 40\%39, 40.

En los últimos tiempos se está evaluando la EMR del NB en sangre periférica (SP), médula ósea (MO) y, si es subsidiario de TASPE, en los productos de aféresis. Los métodos de evaluación más utilizados son la inmunocitología y técnicas de biología molecular, como la RT-PCR, para la detección de marcadores o genes característicos de las células neuroblásticas, como la tirosina hidroxilasa (TH), el GAGE, MA GE y los gangliósidos $\mathrm{GD}_{2}{ }^{16,40-44}$. En el $\mathrm{NB}$ en estadio 4 (alto riesgo), la principal localización metastásica es la médula ósea (MO), por lo que su estudio puede ser útil para evaluar la efectividad de la quimioterapia de inducción y analizada, especialmente después de la cirugía del tumor primario, podría aportar gran información sobre la presencia de micrometástasis. Sin embargo, los métodos citológicos o histológicos estándar no son lo suficientemente sensibles para detectar enfermedad microscópica en MO. La detección molecular de marcadores tumorales mediante RT-PCR constituye un método altamente sensible y específico. Con dicha técnica se procede a la amplificación del ácido ribonucleico mensajero (RN A-m) de la TH, principal enzima involucrada en la síntesis de catecolaminas, y según el grado de su detección, puede ser capaz de predecir recaída a largo plazo en el NB de alto riesg $0^{41,43}$. La inmunocitología, mediante el uso de anticuerpos monoclonales "neuroblastoma específicos"; principalmente los antiGD2, es otro método útil, aunque menos sensible que la RT-PCR para cuantificar la $G D_{2}$ sintetasa, en la detección de células neuroblásticas ocultas en MO y SP16, 42 .

Ciertos estudios han demostrado que la presencia de EMR medida en MO y SP en pacientes con N B Iocalizado mediante el análisis de TH por RT-PCR, se asocia con mayor probabilidad de recaída metastásica tras la extirpación del tumor primario ${ }^{41}$. En el NB de alto riespo, utilizando también el análisis de TH por RT-PCR para detectar EM R, algunos estudios encuentran que la persistencia de la misma en MO tras cuatro meses de comenzar la quimioterapia predice un peor pronóstico (recaída y/ o muerte en los siguientes 24 meses de media, rango de 13-43 meses) en comparación con los que no se observa EMR (no evidencia de enfermedad a los 61 meses de media, rango de 20-76 meses) $)^{43}$.

De especial interés resultan las familias de genes llamadas MAGE, BAGE y GAGE, que codifican distintos antígenos "tumor-asociados" reconocibles por los linfocitos $T$ citotóxicos. Estos antígenos son expresados en células tumorales humanas de distintos tipos histológicos y están silentes en tejidos sanos, excepto en testículo y placenta. Varios grupos han confirmado la expresión de los genes MAGE (MAGE-1, MAGE-2, MAGE3 y M AGE-4) y BAGE en el NB. En un estudio reciente, la detección mediante RT-PCR para GAGE parece tener una sensibilidad mayor que la TH. El gen GAGE tiene una amplia expresión en la mayoria de los N B en estadio 4, y es un marcador sensible y especifico para la detección de micrometástasis de N B en MO y SP16.

Los $\mathrm{GD}_{2}$ son glicoesfingolípidos que contienen ácido siálico y están abundantemente expresados en el NB. Se detectan, no sólo en las células tumorales, sino también en el plasma de los enfermos y por tanto, pueden ser de utilidad para detectar una mínima masa tumoral a distintos niveles. En diferentes estudios se ha observado que la detección de gangliósidos $\mathrm{GD}_{2}$ en $\mathrm{MO}$ por inmunocitologia (utilizando anticuerpos monoclonales anti-GD2) o por RT-PCR, es muy útil para la detección de EMR, viendo que si es positiva se relaciona fuertemente con la progresión de la enfermedad. Los anti-GD2 se fijan a la membrana de la célula tumoral y permiten identificar células aisladas en una suspensión celular ( $p$. ej. muestras de aspirado de MO) 0 en SP. Sin embargo, dicha técnica, precisa de muestras frescas y resulta muy laboriosa; ya que requiere un recuento bajo microscopio. Por el contrario, la detección de EMR midiendo el GD2 por RT-PCR utiliza células mononucleares criopreservadas y puede reproducirse en múltiples ocasiones y para múltiples marcadores. La sensibilidad de detección por inmunocitología del $\mathrm{GD}_{2}$ es de 1 célula tumoral por 100.000 células sanas ( $1 \mathrm{X}$ $\left.10^{-5}\right)$, mientras que por RT-PCR es de 1 por 1.000 .000 (1 $\left.\times 10^{-6}\right)$. La síntesis de los $\mathrm{GD}_{2}$ depende de la enzima $G D_{2}$ sintetasa, cuyo RNA-m supone un potencial marcador tumoral de EMR analizada mediante RT-PCR. El papel de la monitorización de la $G_{2}$ sintetasa resulta de gran utilidad debido a que el $\mathrm{GD}_{2}$ se expresa homogéneamente en todos los estadios del N B. Sus niveles se correlacionan muy bien con el número de células 
GD2 positivas medidas por inmunocitología. Su densidad en los neuroblastos es alta $(5-10 \times 1.000 .000$ moléculas/ célula) y su antígeno glicoesfingolípido disminuye tras el tratamiento con anticuerpos monocionales anti-G $\mathrm{D}_{2}{ }^{42}$.

La inmunoterapia con el anticuerpo monoclonal quimérico anti-G $\mathrm{D}_{2}$ es una modalidad terapéutica utilizada en algunos NB de alto riesgo, cuya efectividad ha sido demostrada y con una toxicidad minima. El anti$\mathrm{GD}_{2}$ reconoce y se une al gangliósido GD2 sobre los neuroblastos e induce la muerte de las células tumorales mediante lisis citotóxica complemento-dependiente y lisis citotóxica anticuerpo-dependiente ${ }^{45-47}$. Diversos trabajos han demostrado que la monitorización mediante RT-PCR de la GD2 sintetasa en MO es un muy útil y precoz marcador para evaluar la eficacia terapéutica de la inmunoterapia con anti-G $\mathrm{D}_{2}{ }^{46,47}$.

Como ya es sabido, las altas dosis de quimioterapia y posteriormente el rescate con TASPE son ampliamente usados en el tratamiento de pacientes con N B de alto riesgo. La reinfusión de células madre (CD34+) de sangre periférica, recolectada por leucoaféresis y contaminada con la presencia de EMR, se ha asociado con recaída postrasplante. Hasta el $77 \%$ de los pacientes con RN A-m para TH presente en los productos de aféresis recaen comparados con el $44 \%$ que fueron negativos, por lo que se sugiere que la presencia de EMR en los productos de aféresis puede jugar un papel determinante en el desarrollo de las recaídas. La detección de células tumorales en muestras de precursores CD34 + de sangre periférica, puede reflejar el estado de una enfermedad avanzada. No obstante, el riesgo de contaminación con células tumorales es más reducido si el autotrasplante se realiza con progenitores hematopoyéticos de sangre periférica comparado con los procedentes de médula ósea ${ }^{44}$. Hoy en día, en estudios abiertos, la disponibilidad de mediciones más especificas y sensibles para la evaluación de la EMR en $M O$ y SP del NB, tienen por objetivo determinar con gran precisión la limpieza relativa de los precursores autólogos, el tiempo apropiado de criopreservación, la eficacia de las técnicas de purgado (purging), así como la eficacia de la terapia mieloablativa y de otros tratamientos adyuvantes ${ }^{46,48-50}$.

\section{Detección de EMR en los rabdomiosarcomas}

En todos los sarcomas de partes blandas en general, y en los rabdomiosarcomas (RMS) en particular, el principal factor pronóstico para la supervivencia a largo plazo, es la presencia de enfermedad diseminada en el momento del diagnóstico. Los pacientes en los que tras finalizar la terapia persiste 0 reaparece EM $R$ en MO y/ o en SP, presentan recaída clínica de forma invariable ${ }^{51}$.

Mediante la técnica de RT-PCR se pueden analizar los productos de fusión específicos de los RMS alveolares en el tumor primario y otros tejidos. Los más frecuentes son:

- el PAX3-FKHR, por la traslocación t $(2,13)$ (q35;q14). Está presente en el $80-90 \%$ de los casos y se suele asociar a enfermedad diseminada en el momento del diagnóstico.

- el PAX7-FKHR, por la $t(1 ; 13)(p 36 ; q 14)$. Presente en el $10-20 \%$ de los casos y asociado a la presentación en pacientes más jóvenes, tendencia a localizarse en las extremidades y ausencia de metástasis en el momento del diagnóstico.

La presencia de estas traslocaciones no sólo se aplica al diagnóstico diferencial del RMS, sino a su influencia sobre el pronóstico y a la detección de EMR, estadificación, evaluación del tratamiento y monitorización de recidivas ${ }^{52}$

Las técnicas de RT-PCR ofrecen ventajas significativas sobre las técnicas morfológicas para la detección de enfermedad micrometastásica en la MO en este tipo de tumores. Además, los pacientes en los que se demuestra la existencia de productos de fusión en el tumor primario, presentan una sensibilidad del $100 \%$. A unque la detección de PAX3-FKHR o PAX7-FKHR en MO se asocia significativamente a una menor supervivencia a largo plazo, queda aún por dilucidar el significado clínico de la evidencia de micrometástasis detectada por RT-PCR en ausencia de afectación morfológica. A pesar de que el $53 \%$ de los pacientes con EMR positiva evolucionan a enfermedad diseminada, frente a un $14 \%$ de los que la tenían negativa, son necesarios más estudios y con un número mayor de pacientes que permitan reestudiar y diseñar nuevas opciones terapéuticas en función de los hallazgos moleculares ${ }^{51-53}$.

Los RMS embrionarios, que además son el tipo de sarcoma de partes blandas más frecuente en la infancia, carecen de traslocaciones específicas. Recientemente se ha sugerido que la detección por PCR del receptor de la acetilcolina fetal (AChR) en este tipo de tumores, es mucho más especifico y sensible que la detección, también por PCR, de la proteína MyoD1. De confirmarse este hallazgo, el AChR constituiría en el futuro un marcador prometedor en la detección de EM R en el RMS $\sin$ traslocaciones tumorales específica $s^{54}$.

\section{Detección de EMR en la familia de tumores de Ewing (sarcoma de Ewing/ pPNET)}

La familia de tumores de Ewing incluye a los sarcomas de Ewing (SE) y los tumores neuroectodérmicos 
primitivos originados a nivel periférico (pPNET). Los tumores de Ewing se presentan habitualmente en el hueso $(87 \%)$ y con menor frecuencia a nivel extraóseo. Las características morfológicas y genéticas de los SE de origen óseo, así como los de origen en tejidos blandos, son muy similares a las de los pPNET, por lo que actualmente se consideran como distintas expresiones de una misma entidad ${ }^{55}$.

Más del $85 \%$ de los tumores de Ewing se caracterizan por una traslococión $\mathrm{t}(11 ; 22)(\mathrm{q} 24 ; \mathrm{ql} / 2)$ en muestras del tumor al diagnóstico, dando lugar al oncogén de fusión FLI1-EW S. También pueden encontrarse algunas de las variantes de la $t(11 ; 22)$, como la $\mathrm{t}(21 ; 22)(q 22 ; q 12)$ que expresa el gen ERG-EW S, presente hasta en un $14 \%$ de los casos. Por lo tanto, en el $81-96 \%$ de los tumores de Ewing puede detectarse algún producto de fusión en el tumor primario, siendo estos casos los subsidiarios del estudio y monitorización de la EMR en MO y/ o SP. Se puede analizar por FISH y RT-PCR el FLI1-EW S o el ERG-EW S en muestras de tumor, MO y SP al diagnóstico, durante el tratamiento y tras finalizar el mismo. El estudio de estos genes de fusión mediante RT-PCR es capaz de detectar 1 célula tumoral dc Ewing entre 106 células normales 51 , 53, 55, 56.

En estudios recientes se ha intentado relacionar la presencia de los productos de fusión característicos de $\mathrm{SE} / \mathrm{pPN} \mathrm{ET}$ en MO en el momento del diagnóstico con el pronóstico del paciente, con resultados dispares entre diferentes grupos. Aunque el factor pronóstico más importante en los tumores de Ewing es la existencia de diseminación metastásica en el momento del diagnóstico, están en estudio otras variables como el tipo de producto de fusión que presentan, fruto de las combinaciones de los exones de ambos genes entre los que se produzca la fusión. Dentro del grupo de oncogenes FLI1-EW S, la fusión más frecuente es la FLI1-EW S tipo 1 (entre el exón 6 del gen FLl y el exón 7 del gen EW S). En un estudio multicéntrico realizado a partir de muestras de 120 tumores de Ewing, se ha podido demostrar que los portadores de esta mutación presentan mayor supervivencia que el resto ${ }^{56}$.

Se ha observado la presencia de EMR en SP entre 2 y 36 meses antes de que tenga lugar la recaída clínica, lo que demuestra la utilidad de su monitorización en el seguimiento evolutivo ${ }^{57}$. La presencia de micometástasis tras la finalización del tratamiento tiene también un significado pronóstico, ya que también se asocia con recaida. N o obstante, se ha visto una baja incidencia de EMR positiva en los productos de aféresis, obtenidos para TASPE, en pacientes con tumores de Ewing de alto ries$g 0^{58}$. Hacen falta más estudios para crear estrategias terapéuticas que logren prevenir la recaída tumoral en los casos de SE/ pPNET que presenten EM R positiva ${ }^{56,59,60 .}$

\section{Conclusiones}

1) La detección de EMR en las leucemias agudas infantiles (ya sea por medio de técnicas de citometría de flujo y/ o estudios moleculares), siempre y cuando se realice de forma seriada durante el curso del tratamiento, puede predecir recaida; sobre todo si dicha EMR se detecta tras los ciclos de consolidación. Estas técnicas también pueden utilizarse y ayudar al estudio de EMR en niños con LAN L.

2) La monitorización de EMR postrasplante de progenitores hematopoyéticos en niños con LA L es de gran importancia clínica, pues nos ayuda a predecir la posibilidad de recaída, hecho que ensombrece mucho el pronóstico de estos pacientes.

3) Es necesario acumular la experiencia derivada de protocolos terapéuticos que incluyan la detección de EMR en la estratificación de los pacientes, para poder concluir que ello influya en elevar las ya de por sí altas tasas curativas de la LA L infantil.

4) La detección de ciertos marcadores como la $\mathrm{TH}$ por RT-PCR y el $G_{2}$ por inmunocitología o por RTPCR en médula ósea, sangre periférica y productos de aféresis, son muy útiles para la detección de EMR en el neuroblastoma durante el tratamiento y tras la finalización del mismo. La positividad de éstos se correlaciona fuertemente con una progresión futura de la enfermedad y en un futuro próximo serán de utilidad para optimizar su manejo terapéutico.

5) La alta sensibilidad y especificidad de la RT-PCR para detectar trascripciones específicas en los rabdomiosarcomas alveolares y en la familia de tumores de Ewing, la hace una técnica útil en la detección y el seguimiento de EM R en este tipo de neoplasias.

6) Hacen falta más estudios para poder aclarar las implicaciones pronósticas y sobre todo la terapia a a plicar a las micrometástasis de estos tumores sólidos, detectadas únicamente por técnicas moleculares.

Correspondencia:

Dr. R. López Almaraz

Servicio de Pediatría

(Unidad de 0 ncohematalogía Pediátrica)

Hospital Universitario de Canarias

Carretera $0 \mathrm{fra}, \mathrm{s} / \mathrm{n}$

E-38320 La Laguna (Santa Cruz de Tenerife) E-mail: ritxil@comtf.es 


\section{Bibliografía}

1. Ravindranath $Y$. Recent advances in pediatric acute lymphoLlastic and myeloid leucemia. Curr 0 pin 0 ncol 2003; 15:23-35.

2. Bernard F, A erts I, Margueritte G, A struc J. M icrometastases in pediatric oncology. Bull Cancer 2001; 88: 57780 .

3. Nichols KE, Li FP, Haber DA, Diller L. Childhood cancer predisposition: applications of molecular testing and future implications. J Pediatr 1998; 132:389-97.

4. Ganjavi H, Malkin D. Genetics of childhood cancer. Clin Orth 2002: 75-87.

5. Look AT, Kirsch IR. Molecular basis of childRood cancer. En: Pizo PA, Poplack DG (eds.). Principles and practice of Pediatric 0 ncology. 4th ed. Philadelphia: Lippincott W illiams \& W ilkins; 2002:45-87.

6. Rubnitz JE, Crist W M. Molecular genetics of childRood cancer: Implications for patRogenesis, diagnosis, and treatment. Pediatrics 1997; 100:101-8.

7. Martínez JA, Castel V, García-Conde J. Citogenética molecular del cáncer infantil: aplicociones clinicas. Med Clin IBarc) 1998; 111:389-97.

8. Harrison CJ. The management of patienis with leukemia: The roie of cytogenetics in this molecular area. $\mathrm{Br} J \mathrm{Hae}$ matol 2000; 108:19-30.

9. Sievers EL, Radich JP. Detection of minimal residual disease in acute leuPemia. Curr 0 pin Hematol 2000; 7:2126.

10. Coustan-Smith E, Sancho J, Hancock ML, Boyett JM, BeLm FG, Raimondi SC et al. Clinical importance of minimal residual disease in childhood acute leukemia. Blood 2000; 96:2691-6.

11. Schrappe M. Prognostic factors in childhood acute lymphoblastic leukemia. Indian J Pediatr 2003; 70:817-24.

12. Pui $\mathrm{CH}$, Campana D. N ew definition of remission in childRood acute lymphoblastic leukaemia. Leukemia 2000; 14:783-5.

13. Coustan-Smith E, Sancho J, Behm FG, Hancock M L, Razouk Bl, Ribeiro RC et al. Prognostic importance of measuring early clearance of leuRemic cells by flow cytometry in childhood acute limphoblastic leukaemia. Blood 2002; 100: 52-8.

14. Dworzak MN, Froschl G, Priniz D, Mann G, Potschger $U$, Mublegger $N$ et al. Prognostic significance and modolities of flow cytometric minimul residual disease detection in childRood acute lymphoLlastic leuLaemia. Blood 2002; 99:1952-8.

15. Bjorklund E, Mazur J, Soderhall S, Porwit-M acDonald A. Flow cytometric follow-up of minimal residual disease in bone marraw gives prognostic information in children with acute Iymphoblastic leukaemia. Leukemia 2003; 17:138-48.

16. Cheung IY, Cheung NK. Detection of microscopic disease: comparing histology, inmunocytology, and RTPCR of tyrosine hydroxylase, GAGE, and MAGE. Med Pediatr O ncol 2001; 36:210-2.

17. Silverman LB, Sallan SE. N ewly diagnosed childhood acute lymphoblastic leukemia: update on prognostic factors and treatment. Curr 0 pin Hematol 2003; 10:290-6.

18. Borowit MJ, Pullen DJ, Shuster JJ, Viswanatha D, Montgomery $\mathrm{K}, \mathrm{W}$ illman $\mathrm{CL}$ et al. M inimal residual disease detection in childhood precursor-B-cell acute lymphoblastic leukaemia: relation to other risk factors. A Children s Oncology Group syudy. Leukemia 2003; 17:1566-72.

19. Pine SR, Moy FH, W iemels JL, G rill RK, Levendoglu-Tugal O, O zkaynak MF et al. Real-time quantitative PCR: standardized detection of minimal residual disease in pediatric acute lymphoblastic leukemia. J Pediatr Hematol O ncol 2003; 25:103-8.

20. Viehmann S, Teigler-Schlegel A, Bruch J, Langebrake C, Reinhardt $D$, Harbott J. Monitoring of minimal residual disease (MRD) by real time-quantitative reverse transcription PCR (RQ-RT-PCR) in childRood acute myeloid leukaemia with AML1/ ETO rearrangement. Leukemia 2003; 17:1130-6.

21. Morschhauser F, Cayuela JM Martini S, Baruchel A Rousselot $P$, Socie $G$ et al. Evaluation of minimal residual disease using reverse-transcription polymerase chain reaction in $t(8 ; 21)$ acute myeloid leukemia: a multicenter study of 51 patients. J Clin 0 ncol 2000; 18:788-94.

22. Guerrasio A, Pilatrino C, De Micheli D, Cilloni D, Serra A, Gottardi $E$ et al. A ssessment of minimul residual disease (MRD) in CBF(/ M YH11-positive acute myeloid leukemias by qualitative and quantitative RT-PCR amplification of fusion transcipts. Leukemia 2002; 16:1176-81.

23. Buonamici S, Ottaviani E, Testoni N, Montefusco V, Visani $G$, Bonifazi $F$ et al. Real-time quantitation of minimal residual disease in inv(16)-positive acute myeloid leukemia may indicate risk for clinical relapse and may identify patients in a curable state. Blood 2002; 99:4439.

24. Eckert C, Biondi A, Seeger K, Cazaniga G, Hartmann R, Beyermann $B$ et al. Prognostic value of minimal residual disease in relapsed childhood acute lymphoblastic leuka emia. Lancet 2001; 358:1239-41.

25. W illemse MJ, Seriu T, Hettinger K, d A niello E, Hop W C, Panzer-G rumayer ER et al. Detection of minimal residual disease identifies differences in treatment response between T-ALL and precursor B-ALL. Blood 2002; 99:4386-93.

26. Nyvold C, Madsen HO, Ryder LP, Seyforth J, Svejgacrd $A$, Clausen $N$ et al. Precise quantification of minimal residual disease at day 29 allows identification of children with acute lymphoLlastic leukaemia and an excellent outcome. Blood 2002; 99:1253-8.

27. Marskall GM, Haber M, Kwan E, Zhu L, Ferrara D, 
Chengyuan $X$, et al. Importance of minimul residual disease testing during the second year of therapy for children with acute Iymphoblastic leukemia. J Clin O ncol 2003; 21:704-9.

28. Subirá $D$, Castanon $S$, Roman A, A ceituno $E$, JiménezGarofono $C$, Jiménez $A$ et al. Flow cytometry and the study of central nervous disease in patients with acute leuka emia. Br J Haematal 2001; 112:381-4.

29. de Haos V, Vet RJ, Verhagen $0 J$, Kroes W, van den Berg $H$, van der Schoot CE. Early detection of central nervous system relapse by polymerase chain reaction in children with B-precursor acute lymphoblastic leukemia. Ann Hematol 2002; 81:59-61.

30. Coustan-Smith E, Ribeiro RC, Rubnit JE, Razouk BI, Pui $\mathrm{CH}$, Pounds $\mathrm{S}$ et al. Clinical significance of residual disease during treatment in childhood acute myeloid leukaemia. Br J Haematal 2003; 123:243-52.

31. Goulden N, Bader P, Van Der Velden V, Moppett J, Schilham M, Masden HO, et al. Minimal residual disease prior to stem cell transplant for childRood acute lymphoblastic leuLa emia. Br J Haematol 2003; 122:24-9.

32. Schneider M, Hettinger K, Matthes-Martin S, Konrad M, Peters C, Gadner H et al. Influence of transplantation regimen on prognostic significance of high-level minimal residual disease before allogenic stem cell transplantation in children with ALL. Bone Marrow Transplant 2001; 28:1087-9.

33. Sánchez J, Serrano J, Gómez P, Martínez F, Martín C, Madero $L$ et al. Clinical value of inmunological monitoring of minimul residual disease in acute lymphoblastic leukemia aker allogenic transplantation. $\mathrm{Br} J$ Haematol 2002; 116: 686-94.

34. Uzunel M, Jaksch M, Mattsson J, Ringden O. M inimal residual detection aker allogenic stem cell transplantation is correlated to relapse in patients with acute lymphoblastic leukaemia. Br J Haematol 2003; 122:788-94.

35. Palacio $C$. Enfermedad mínima residual en la leucemia aguda linfoblástica del niño. Haematológica (ed. Esp.) 2002; 87 (suppl.1):267-9.

36. Rubnitz JE, Pui $\mathrm{CH}$. Recent advances in the treatment and understanding of childhood acute lymphoblastic leuka emia. Cancer Treat Rev 2003; 29:31-44.

37. Moppett J, BurLe GA, Steward CG, O akhill A, Goulden $\mathrm{NJ}$. The clinical relevance of detection of minimal residual disease in childhood acute lymphoblastic leukaemia. J Clin Pathol 2003; 56:249-53.

38. Campbell PJ, Morley AA. Modelling a minimal residual disease-based treatment strategy in childhood acute lymphoblastic leukaemia. Br J Haematol 2003; 122:308.

39. Brodeur GM, Maris JM. N euroblastoma. En: Pizo PA, Poplack DG (eds.). Principles and practice of Pediatric O ncology. 4th ed. Philadelphia: Lippincott W illiams \& W ilkins; 2002:895-937.

40. Fukuda M, Miyalima Y, Miyashita Y, Horibe K. Disease outcome may be predicted by molecular detection of mi- nimal residual disease in bone marrow in advanced neuroblastoma: a pilot study. J Pediatr Hematol O ncol $2001 ; 23: 10-3$

41. Shono K, Taliri T, Fujii Y, Suita S. Clinical implications of minimul disease in bone marrow and peripheral blood in neuroblastoma. J Pediatr Surg 2000; 35:1415-20.

42. Cheung IY, Cheung N K. Q uantitation of marrow disease in neuroblastoma by real-time reverse transcription-PCR. Clin Cancer Res 2001; 7:1698-705.

43. Horibe K, Fukuda M, Miyajima $Y$, Matsumoto K, Kondo $M$, Inaba J et al. O utcome prediction by molecular detection of minimal residual disease in bane marrow for advanced neuroblastoma. Med Pediatr 0 ncol 2001; 36:203-4.

44. Burchill $S A$, Kinsey $S E$, Picton $S$, Roberts $P$, Pinkerton $C R$, Selby $P$ et al. Minimal residual disease at the time of peripheral blood stem cell harvest in patients with advanced neuroLlastoma. Med Pediatr O ncol 2001; 36:21 39.

45. Raffaghello $L$, Marimpietri $D$, Pagnan $G$, Pastorino $F$, Cosimo $E$, Brignole $C$ et al. Anti-GD2 monoclonal antibody immunotherapy: a promising strategy in the prevention of neuroLlastoma relapse. Cancer Lett 2003; 197:205-9.

46. Cheung IY, Lo Piccolo MS, Kushner BH, Kramer K, Cheung NK. Q uantitation of GD2 syntRase mRNA by realtime reverse transcriptase polymerase chain reaction: clinical utility in evaluating adjuvant therapy in neuroblastoma. J Clin O ncol 2003; 21:1087-93.

47. Cheung IY, Lo Piccolo MS, Kushner BH, Cheung NK. Early molecular response of marrow disease to biologic therapy is highly prognostic in neuroblastoma. J Clin Oncol 2003; 21:3853-8.

48. Faulkner LB, Garaventa A, Paoli A, Tintori V, Tamburin $A$, Lacitignola $L$ et al. In vivo cytoreduction studies and cell sorting-enhanced tumor-cell detection in high-risk neuroblastoma patients: implications for leukopheresis strategies. J Clin O ncol 2000; 18:3829-36.

49. Handgretinger $R$, Lang $P$, Ihm K, Schumm M, Geiselhart $A$, Koscielnick $E$ et al. Isolation and transplantation of highly purified autologous peripReral CD34 (+) progenitor cells: purging efEicocy, hematopoietic reconstitution and long-term outcome in children with high-risk neuroblastoma. Bone Marrow Transplant 2002 ; 29:731-6.

50. Cheung IY, Lo Piccolo MS, Collins N, Kushner BH, Cheung NK. Q uantitation of GD2 synthase mRNA by realtime reverse transcription-polymerase chain reaction: utility in bone marrow purging of neuroblastoma by anti-GD2 antiCody 3F8. Cancer 2002; 94:3042-8.

51. W illele F, Sturm JW. M inimal residual in soft-tissue sarcomas. Semin Surg O ncol 2001; 20:294-303.

52. Kelly KM, W ormer RB, Barr FG. Minimal disease detection in patients with alvealar rFabdomyosarcoma using a reverse transcriptase-polymerase chain reaction method. Cancer 1996; 78:1320-7.

53. A thale UH, Shurtlehf SA, Jenkins JJ, Poquette CA, Tan 


\section{R. López Almaraz y cols.}

$M$, Downing JR et al. Use of reverse transcriptase polymerase chain reaction for diagnosis and stoging of alvealar rFabdomyosarcoma, Ewing sarcoma family of tumors, and desmoplastic small round cell tumor. J Pediatr Hematol O ncol 2001; 23:99-104.

54. Gattenloehner S, Dockhorn-Dworniczak B, Leuschner I, Vicent A, Muller-Hermelink HK, Marx A. A comparison of MyoD1 and fetal acetylcholine receptor expression in childhood tumors and normal tissues: implications for the molecular diagnosis of minimul disease in rhabdomyosarcomas. J Mol Diagn 1999; 1:23-31.

55. Ginsserg JP, W oo SY, Johnson ME, Hicks MJ, Horowit ME. Ewing's sarcoma family of tumors: Ewing s sarcoma of bone and soh tissue and the peripReral primitive neuroectodermul tumors. En: Pizzo PA, Poplack DG (eds.). Principles and practice of Pediatric 0 ncology. 4th ed. Philadelphia: Lippincott W illiams \& W ilkins; 2002:973. 1016.
56. De Alava E, Pardo J. Ewing tumor: tumor biology and clinical applications. Int J Surg Pathol 2001; 9:7-17.

57. De Alava E Lozano MD, Patiño A, Sierrasesumaga L, Pardo-Mindán FJ. Ewing family tumors: potencial prognostic value of reverse-transcriptase polymerase chain reaction detection of minimul residual disease in peripheral biood simples. Diagn Mol Pathol 1998; 7:152-7.

58. Fischmeister $G$, ZouSek A, Jugovic D, W itt V, Ladenstein $R$, Fritsch $G$ et al. Low incidence of molecular evidence for tumour in PBPC harvesis from patients with high risk Ewing tumors. Bome Marrow Transplant 1999; $24: 4059$

59. Rodríguez-Galindo C, Spunt SL, Pappo AS. Treatment of Ewing sarcoma family of tumors: current status and 0 utlook for the future. Med Pediatr 0 ncol 2003; 40:276-87.

60. Kovar H. Ewing tumor biology: perspectives for innovative treatment approaches. Adv Exp Med Biol 2003; $532: 27-37$ 